Check for updates

Cite this: RSC Adv., 2017, 7, 41919

Received 4th May 2017

Accepted 18th August 2017

DOI: $10.1039 / c 7 r a 05042 a$

rsc.li/rsc-advances

\section{Surface functionalization of superparamagnetic nanoparticles by an acid-liable polysaccharide- based prodrug for combinatorial monitoring and chemotherapy of hepatocellular carcinoma $\uparrow$}

\author{
Chaoping Fu, (D) $+^{a}$ Rui-Meng Yang, ${ }^{\text {bc }}$ Li Wang, ${ }^{b}$ Nan-nan Li, ${ }^{a}$ Meng Qi, ${ }^{b}$ \\ Xiang-dong $\mathrm{Xu}^{\mathrm{bc}}$ Xin-hua Wei, ${ }^{\text {bc }}$ Xin-Qing Jiang ${ }^{\star \mathrm{bc}}$ and Li-Ming Zhang ${ }^{\star a}$
}

\begin{abstract}
For combinatorial monitoring and chemotherapy of hepatocellular carcinoma, the macromolecular prodrug based on hyaluronic acid and doxorubicin hydrochloride was prepared by an acid-labile hydrazone linkage, and then conjugated with amine-modified iron oxide nanoparticles by a carbodiimide mediated coupling reaction. The resultant hybrid nanoparticles have the characteristics of good water dispersibility, superparamagnetic property and high magnetic relaxivity for magnetic resonance imaging. In particular, they demonstrated significant cellular uptake and specific accumulation in human hepatocellular liver carcinoma HepG2 cells due to specific biological recognition of hyaluronic acid. In addition, in vitro cumulative release of doxorubicin hydrochloride from the resultant hybrid nanoparticles was significantly accelerated at a mildly acidic $\mathrm{pH}$ of 5.0-6.0 when compared to its release at the physiological $\mathrm{pH}$ of 7.4 , suggesting the $\mathrm{pH}$-responsive feature of this conjugated prodrug for effective chemotherapy of hepatocellular carcinoma. In vivo antitumor efficacy of the mice further confirmed the effect.
\end{abstract}

\section{Introduction}

Hepatocellular carcinoma is one of the most common causes of cancer, and its incidence is still increasing worldwide. Despite modern advancements in diagnosis, prevention, and therapy, these diseases still affect millions of patients and impair the patients' quality of life. Thus, it is a significant task to find effective methods to monitor and treat these diseases. It would be ideal if one platform could be used for both monitoring and treatment. In this regard, the theranostics approach has been developed in the past years. Actually, there is an urgent need to find such eligible platforms, but it is still a grave challenge. With the development of novel nanoparticle delivery systems, the priority is to design and synthesize new nanoparticles that, meanwhile, are capable of treating and detecting a disease via binding to effective drugs, probes and specific biomarker/target of the disease.

${ }^{a}$ PCFM Lab, GDHPPC Lab, School of Materials Science and Engineering, Sun Yat-Sen University, Guangzhou 510275, China.E-mail: ceszhlm@mail.sysu.edu.cn

${ }^{b}$ Department of Radiology, Guangzhou First People's Hospital, Guangzhou Medical University, Guangzhou, 510180, China.E-mail: jiangxinqing888@163.com

${ }^{c}$ Department of Radiology, The Second Affiliated Hospital, South China Univeristy of Techology, Guangzhou, Guangdong, 510640, China

$\dagger$ Electronic supplementary information (ESI) available. See DOI: 10.1039/c7ra05042a

\$ These authors contributed equally to this work.
There are several types of materials used in theranostics. ${ }^{1-5}$ This work focus on the theranostic materials that combining both chemotherapy and magnetic resonance imaging (MRI). Traditionally chemotherapy for cancer treatment is facing tremendous challenges due to the drug resistance of cancer cells and systemic side effects which nonspecific action on both the tumor and normal cells. Recent advances have shown that the situation may be improved by using a polymer-based material for theranostics, such as liposome, micelle, vesical and so on. There are two main strategies to incorporate anticancer drugs into polymeric nanocarriers. The first one is physical encapsulation, which using amphiphilic block copolymers assembled into micellar or polymersomes structures. ${ }^{6-9}$ Unfortunately, these previous researchers suffer from a relatively complicated preparation process or the drugs may leak out of the nanomedicines. ${ }^{10}$ The second one adopts chemical conjugation to load drugs into polymeric nanocarriers. ${ }^{\mathbf{1 1 , 1 2}}$

In this work, we carried out for the first time the facile conjugation of hyaluronic acid and doxorubicin $\mathrm{pH}$ responsive prodrug (HA-DOX) with superparamagnetic iron oxide nanoparticles (SPIONs) by a carbodiimide mediated coupling reaction. It is known that hyaluronic acid (HA) is a naturally occurring glycosaminoglycan with specific biological recognition to human tumor cells overexpressing CD44 HA receptors, ${ }^{13}$ and this study set out to evaluate the MRI ability and chemotherapy effect of SPIO-HA-DOX for targeted 
liver cancer to investigate its possibility to be used as a theranostic agent.

\section{Experimental section}

\subsection{Materials}

Amine-functionalized SPIOs ( $\mathrm{NH}_{2}$-SPIOs) was synthesized according to the method of Yang et al. ${ }^{14}$ as reported in our previous publication. 1-(3-Dimethylaminopropyl)-3-ethylcarbodiimide hydrochloride (EDC), $N$-hydroxysuccinimide (NHS), doxorubicin hydrochloride (DOX), adipic acid dihydrazide (ADH), 1-hydroxybenzotriazole (HOBt) and fluorescein isothiocyanate (FITC) were purchased from Aladdin Reagent Database Inc. (Shanghai, China). Hyaluronic acid (HA, MW $320 \mathrm{kDa}$ ) was purchased from Freda Biochem Co., Ltd. (Shandong, China). 3-Aminopropyltrimethoxysilane (APTMS) was purchased from Sigma-Aldrich (St. Louis, MO). Phosphatebuffered saline (PBS; $10 \mathrm{mM}$, pH 7.4), fetal bovine serum (FBS, 10\%), Dulbecco's modified Eagle's medium (DMEM) and Roswell Park Memorial Institute medium (RPMI) were purchased from Hyclone/Thermo Scientific. All other reagents were of analytical grade and were used without further purification.

\subsection{Cell culture and animals}

HepG2 cells (HB 8065; American Type Culture Collection (ATCC), Rockville, MD) were cultured in DMEM supplemented with $10 \%$ of FBS, antibiotics $\left(100 \mu \mathrm{g} \mathrm{mL} \mathrm{m}^{-1}\right.$ streptomycin and $100 \mathrm{U} \mathrm{mL}^{-1}$ penicillin) at $37{ }^{\circ} \mathrm{C}$ in a humidified atmosphere with $5 \%$ of $\mathrm{CO}_{2}$. Cells were both harvested by the use of trypsin and re-suspended in a fresh complete medium before plating.

This study was performed in strict accordance with the Guide to the Care and Use of Experimental Animals by the National Research Council (1996, United States). All experimental and animal-handling procedures were approved by the Sun Yat-sen University Cancer Center Ethics Committee on the Use of Live Animals in Teaching and Research (Laboratory Animal Center of Sun Yat-sen University, Guangzhou, China).

\subsection{Preparation of HA-ADH conjugates}

Adipic acid dihydrazide modified HA (HA-ADH) was synthesized by coupling primary carboxyl group of sodium hyaluronic acid with amino group of adipic acid dihydrazide..$^{15}$ First, $0.5 \mathrm{~g}$ HA was dissolved in $100 \mathrm{~mL}$ distilled water at a concentration of $5 \mathrm{mg} \mathrm{mL}{ }^{-1}$. A total of $2.613 \mathrm{~g}$ of ADH $(15 \mathrm{mmol})$ was added to the solution of HA. Then, $0.96 \mathrm{~g}$ EDC ( $5 \mathrm{mmol})$ and $0.675 \mathrm{~g} \mathrm{HOBt}$ (5 mmol) were added dropwise. This mixture was stirred at ambient temperature for $24 \mathrm{~h}$ ( $\mathrm{pH}$ 6.8). The resulting solution was dialyzed (MWCO 3000) exhaustively for 3 days against water to remove unreacted agents. Finally, the products (HA-ADH) were obtained by lyophilization and stored at $4{ }^{\circ} \mathrm{C}$ until further use. Yield: $85.6 \% .{ }^{1} \mathrm{H}$ NMR $\left(300 \mathrm{MHz}, \mathrm{D}_{2} \mathrm{O}\right): \delta 2.29(2 \mathrm{H}$, $\left.\mathrm{m},-\mathrm{NHNHCOCH}_{2}-\right), 2.12\left(2 \mathrm{H}, \mathrm{m},-\mathrm{CH}_{2} \mathrm{NHNH}_{2}\right), 1.90(15 \mathrm{H}, \mathrm{bs}$, $\mathrm{CH}_{3} \mathrm{C}(\mathrm{O})$ ), $1.67-1.36\left(4 \mathrm{H}, \mathrm{m},-\mathrm{CH}_{2} \mathrm{CH}_{2}-\right.$ ), 3.2-3.9 (protons of $\mathrm{HA}$ disaccharide unit ( $\mathrm{H}-2, \mathrm{H}-3, \mathrm{H}-4, \mathrm{H}-5, \mathrm{H}-6)), 4.55(\mathrm{H}-1$ from $\mathrm{N}$ actylglucosamine unit), 4.35 ( $\mathrm{H}-1$ from glucuronic acid). The degree of substitution by ADH was about $24 \%$ determined by digital integration of the NMR signals arising from the anomeric protons of HA and methylene protons of ADH.

\subsection{Synthesis of hyaluronic acid-doxorubicin conjugates}

$0.50 \mathrm{~g}$ HA-ADH conjugates were dissolved in distilled water at the concentration of $10 \%(\mathrm{w} / \mathrm{w})$. The $\mathrm{pH}$ of the reaction mixture was adjusted to 5.0 by addition of acetic acid. Doxorubicin hydrochloride (dissolved in $5 \mathrm{~mL}$ of water) was added dropwise. The mixture was allowed to proceed for $24 \mathrm{~h}$ at $37^{\circ} \mathrm{C}$. After the reaction, the mixture was cooled to room temperature and dropped into $100 \mathrm{~mL}$ of ethanol to precipitate the hyaluronic acid-doxorubicin prodrug. The precipitates were washed with ethanol for twice and dried at $40{ }^{\circ} \mathrm{C}$ under vacuum for $48 \mathrm{~h}$ to obtain the hyaluronic acid-doxorubicin (HA-DOX) conjugates. Yield: $72 \% .{ }^{1} \mathrm{H}$ NMR $\left(500 \mathrm{MHz}, \mathrm{D}_{2} \mathrm{O}\right) \delta 8.2(1 \mathrm{H},-\mathrm{N}-\mathrm{NH}-\mathrm{CO}-)$; 7.8-7.4 $(3 \mathrm{H}, \mathrm{m}$, phenyl $\mathrm{H}), 5.41\left(1 \mathrm{H}, \mathrm{s},-\mathrm{CH}_{2} \mathrm{OH}\right), 4.18(5 \mathrm{H}, \mathrm{s}$, $-\mathrm{CH}-$ of sugar ring and $\left.3 \mathrm{H}, \mathrm{CH}_{3} \mathrm{OAr}\right), 2.29(6 \mathrm{H}, \mathrm{m}$, $\left.-\mathrm{NHNHCOCH}_{2}-\right), 2.16\left(6 \mathrm{H}, \mathrm{m},-\mathrm{CH}_{2} \mathrm{NHNH}_{2}\right), 1.90(36 \mathrm{H}, \mathrm{bs}$, $\left.\mathrm{CH}_{3} \mathrm{C}(\mathrm{O})\right), 1.67-1.36\left(12 \mathrm{H}, \mathrm{m},-\mathrm{CH}_{2} \mathrm{CH}_{2}{ }^{-}\right), 1.1\left(3 \mathrm{H}, \mathrm{m},-\mathrm{CH}_{3}\right.$ of sugar ring), 3.2-3.9 (protons of HA disaccharide unit $(\mathrm{H}-2, \mathrm{H}-3$, $\mathrm{H}-4, \mathrm{H}-5, \mathrm{H}-6)$ ), 4.55 (H-1 from $N$-actylglucosamine unit), 4.35 ( $\mathrm{H}-1$ from glucuronic acid).

\subsection{Synthesis of HA-DOX coated SPIOs}

For the chemical conjugation of amine-functionalized SPIOs with the hyaluronic acid-doxorubicin conjugates (HA-DOX), $150 \mathrm{mg}$ of $\mathrm{NH}_{2}$-SPIOs was first dispersed by sonication in $100 \mathrm{~mL}$ of water. Afterward, EDC (0.3535 g) and NHS (0.1826 g) were then added successively. After that, aqueous solution $(20 \mathrm{~mL})$ of HA-DOX $(450 \mathrm{mg})$ was added dropwise and reacted for $24 \mathrm{~h}$ at room temperature. HA-DOX coated SPIOs (SPIO-HADOX) were collected by applying an external magnetic field and washing with deionized water several times and then centrifuged (1000 rpm, $10 \mathrm{~min})$ to remove the bigger nanoparticles.

\subsection{Characterization techniques}

$5 \mathrm{mg}$ of each sample was dissolved in $0.5 \mathrm{~mL} \mathrm{D}_{2} \mathrm{O}$ and the ${ }^{1} \mathrm{H}$ NMR data was obtained by a Varian Mercury-Plus (300 MHz, USA) spectrometer. The Fourier transform infrared (FTIR) spectra were obtained by a Nexus 670 model FTIR spectrophotometer (Thermo Nicolet, USA). The thermogravimetric analysis (TGA) was carried out by using a TG-209 F1 thermogravimetric thermal analyzer (Netzsch, Germany) in a temperature range of $25-800{ }^{\circ} \mathrm{C}$ with a heating rate of $10^{\circ} \mathrm{C} \mathrm{min}^{-1}$ under nitrogen atmosphere. The dispersion/aggregation state of the nanoparticles in water was performed using a Zeta PALS instrument (Brooken Haven, USA). The samples were dispersed in water with a concentration of $0.1 \mathrm{mg} \mathrm{mL}^{-1}$ before measurements. The $\mathrm{X}$-ray diffraction (XRD) measurements were performed on a D/MAX 2200 VPC X-ray diffractometer (Japan) using Cu KR radiation. The magnetic measurements were done with a superconducting quantum interference device (SQUID) model MPMS Xl-7 magnetometer (USA) with a maximum magnetic field of $1 \mathrm{~T}$ and a sensibility of $10^{-6} \mathrm{emu}$. The transmission 
electron microscopy (TEM) images were obtained on a JEM2010HR transmission electron microscope (Japan). The $T_{2}$ relaxometry was carried out using a 3.0 T MR Analyzing system (Verio, Siemens, Erlangen, Germany). A phenanthroline assay ${ }^{17}$ was used for quantitative assessment of iron content. Phantom MRI of SPIO and SPIO-HA-DOX NPs was carried out at various iron concentration from 0.09 to $0.45 \mathrm{mM}$ using a $T_{2}$ mapping sequence (TR: $1000 \mathrm{~ms}$, TE: 13.8/27.6/41.4/55.2/69.0 ms, flip angle: $180^{\circ}$, slice thickness: $3.0 \mathrm{~mm}$, matrix $444 \times 448$ ).

\subsection{In vitro drug release}

The SPIO-HA-DOX were taken into dialysis bags (MWCO = 3000), and then immerged into $50 \mathrm{~mL}$ tubes containing $20 \mathrm{~mL}$ phosphate buffer saline (PBS, pH 7.4) as the release media. The tubes were incubated in a shaking water bath at $37{ }^{\circ} \mathrm{C}$ during the test. At a predetermined period of the in vitro release experiment, $3.0 \mathrm{~mL}$ of the buffer solution was removed from the tubes and $3.0 \mathrm{~mL}$ fresh buffer solution was added back into the tubes to maintain the same total solution volume. The amount of released DOX was determined by a UV spectrophotometer at the absorbance wavelength of $480 \mathrm{~nm}$. The percentage of cumulative amount of released DOX was determined from the standard calibration curves.

\subsection{Prussian blue staining}

HepG2 cells at a density of $5 \times 10^{5}$ cells per well were seeded onto the 12-well plate and incubated for $24 \mathrm{~h}$. After removing the media, $1 \mathrm{~mL}$ of the DMEM medium containing SPIO or SPIO-HADOX NPs (Fe concentration was fixed to be $40 \mu \mathrm{g} \mathrm{mL}{ }^{-1}$ ) was added and incubated at $37{ }^{\circ} \mathrm{C}$ for another $24 \mathrm{~h}$. After removing the supernatant, the cells were washed with PBS for three times and then were fixed by $1 \mathrm{~mL} 4 \%$ paraformaldehyde solution for $20 \mathrm{~min}$. To each well, $1 \mathrm{~mL}$ of a $1: 1$ mixture of $2 \%$ potassium ferrocyanate trihydrate and 4\% hydrochloric acid (in PBS) was added. The cells were incubated in the dark for $30 \mathrm{~min}$ at $37{ }^{\circ} \mathrm{C}$ causing a deep blue complex, rewashed and then counterstained with nuclear fast red for $5 \mathrm{~min}$. The wells without SPIO or SPIOHA-DOX NPs were used as control. Prussian blue staining images were taken on an Olympus BX51 optical microscope (Olympus Corp, Tokyo, Japan).

\subsection{Fluorescence imaging and flow cytometry}

HepG2 cells were cultured in glass-bottom dishes for $24 \mathrm{~h}$ till total adhesion. An aliquot of DOX solution, HA-DOX and SPIOHA-DOX with final DOX concentration of $5 \mu \mathrm{g} \mathrm{mL}{ }^{-1}$ was added to each well. After $4 \mathrm{~h}$ incubation, the medium was removed and cells were washed with $1 \mathrm{~mL}$ PBS three times to remove NPs not taken up by cells. Then cells were fixed with $4 \%$ paraformaldehyde in PBS at room temperature for $10 \mathrm{~min}$, the wells without NPs were used as blank. Then, cell-associated fluorescence was investigated by a fluorescence microscope (IX71, Olympus, Japan). To quantify the uptake of DOX by the tumor cells, free DOX, HA-DOX and SPIO-HA-DOX with a final DOX concentration of $5 \mu \mathrm{g} \mathrm{mL}{ }^{-1}$ were incubated with HepG2 cells for $4 \mathrm{~h}$, following which the cellular fluorescence intensity was measured by flow cytometers (Guava Easy Cyte ${ }^{\mathrm{TM}}$, Hayward, CA,
USA). The events collected were ten thousand and the data were analyzed. The intracellular DOX level of each sample was presented as the average fluorescence intensity.

\subsection{In vitro cytotoxicity}

Cellular toxicity assays were carried out by MTT (3-(4,5dimethyl-2-thiazolyl)-2,5-diphenyl- $2 H$-tetrazolium bromide) method on HepG2 cells. Briefly, $1 \times 10^{4}$ cells per well were seeded in 96-well plates for $24 \mathrm{~h}$ at $37^{\circ} \mathrm{C}$ prior to drug addition. The cells were incubated with various amounts of free DOX, HA-DOX and SPIO-HA-DOX at the equivalent drug concentration for $0.01,0.1,1,2.5,5,7.5,10,12.5,15 \mu \mathrm{g} \mathrm{mL}{ }^{-1}$. The culture medium without any drug formulations was used as the control. After $24 \mathrm{~h}$ of incubation, the medium was removed and the wells were washed with PBS for two times. $20 \mu \mathrm{L}$ MTT in a concentration of $5 \mathrm{mg} \mathrm{mL}^{-1}$ was added to each well and the cells were incubated for another $4 \mathrm{~h}$ at $37{ }^{\circ} \mathrm{C}$. Finally, formazan crystals were solubilized by $150 \mu \mathrm{L}$ of DMSO and the absorbance was measured with the ELISA reader Thermo Multiscan MK3 at wavelength $490 \mathrm{~nm}$. The relative cell viability (\%) related to the control wells containing cell culture medium was calculated by the following equation:

$$
\text { Cell viability }(\%)=\left(A_{\text {test sample }} / A_{\text {control }}\right) \times 100 \%
$$

where $A_{\text {test sample }}$ is the absorbance of the test sample and $A_{\text {control }}$ is the absorbance of the control.

\subsection{In vitro cytotoxicity}

HepG2 cells, at a density of $1 \times 10^{6}$ cells per $\mathrm{cm}^{2}$, were washed and trypsinized after incubation with SPIO or SPIO-HA-DOX NPs, respectively, at Fe concentrations of $40 \mu \mathrm{g} \mathrm{mL} L^{-1}$ in serum-free DMEM medium at $37{ }^{\circ} \mathrm{C}$ for $24 \mathrm{~h}$. Cells were then mixed with $2 \%$ agarose solution in PBS and scanned under a 3.0 T MRI scanner (Verio, Siemens, Erlangen, Germany) at room temperature. $T_{2}$ mapping sequence (TR: $1000 \mathrm{~ms}$, TE: 13.8/27.6/ 41.4/55.2/69.0 ms, flip angle: $180^{\circ}$, slice thickness: $3.0 \mathrm{~mm}$, matrix $444 \times 448$ ) was used to measure transverse relaxation time. $T_{2}$ values of the various solutions were analyzed by defining regions of interest (ROI) in each test tube.

\subsection{In vivo MR imaging}

Athymic nude mice bearing HepG2 tumors were used for in vivo MR imaging studies. Tumors were inoculated by injecting a suspension of $1 \times 10^{7}$ HepG2 cells in physiological saline (200 $\mu \mathrm{L})$ into the right subcutaneous dorsa of mice. The tumor targeting ability of SPIO-HA-DOX after systemic administration was investigated using a 3.0 T MRI scanner (Verio, Siemens, Erlangen, Germany). MR images were taken prior to the injection of SPIO-HA-DOX and at appropriate time points postinjection. Mice were anaesthetized for imaging with 10\% chloral hydrate by intraperitoneal injection. Then an aqueous dispersion of SPIO-HA-DOX was injected through the tail vein at a dose equivalent to $5 \mathrm{mg}$ Fe per $\mathrm{kg}$. In vivo $T_{2}$-weighted fast spin-echo imaging was performed under the following conditions: $\mathrm{TR}=4500 \mathrm{~ms}$, $\mathrm{TE}=82 \mathrm{~ms}, \mathrm{FOV}=8 \times 8 \mathrm{~cm}^{2}$, section 
thickness $=2 \mathrm{~mm}$ (transverse section) $/ 3 \mathrm{~mm}$ (coronal section). All MRI quantitative analyses were carried out by one radiologist.

\subsection{In vivo anti-tumor efficacy}

The tumor regression effect of SPIO-HA-DOX was investigated in BALB/C nude mice (4-5 weeks old, 15-20 g) inoculated hepatic cancer HepG2 cells $\left(1 \times 10^{7}\right.$ cells per mouse) subcutaneously in the right flank of the mice. One week later, the mice were randomly divided into four groups (4 mice per group), and each group was treated by tail vein injection every four days with one of the following: physiological saline (control); DOX; HA-DOX and SPIO-HA-DOX. The dose of DOX was $2 \mathrm{mg} \mathrm{kg}^{-1}$, respectively. Five injections were performed in the treatment process. Tumor volumes were measured every treatment point. Measurement of tumor size was performed with a caliper in two dimensions, and individual
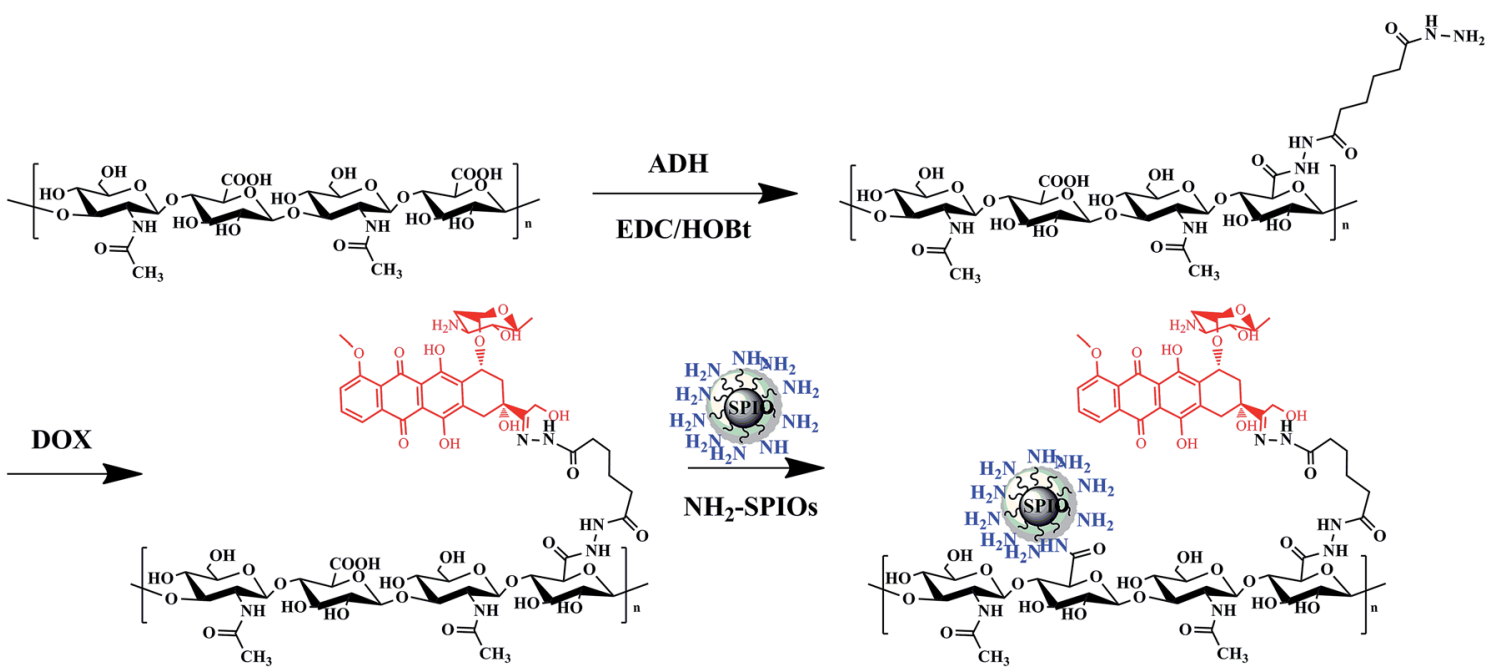

Fig. 1 The synthesis of SPIO-HA-DOX.
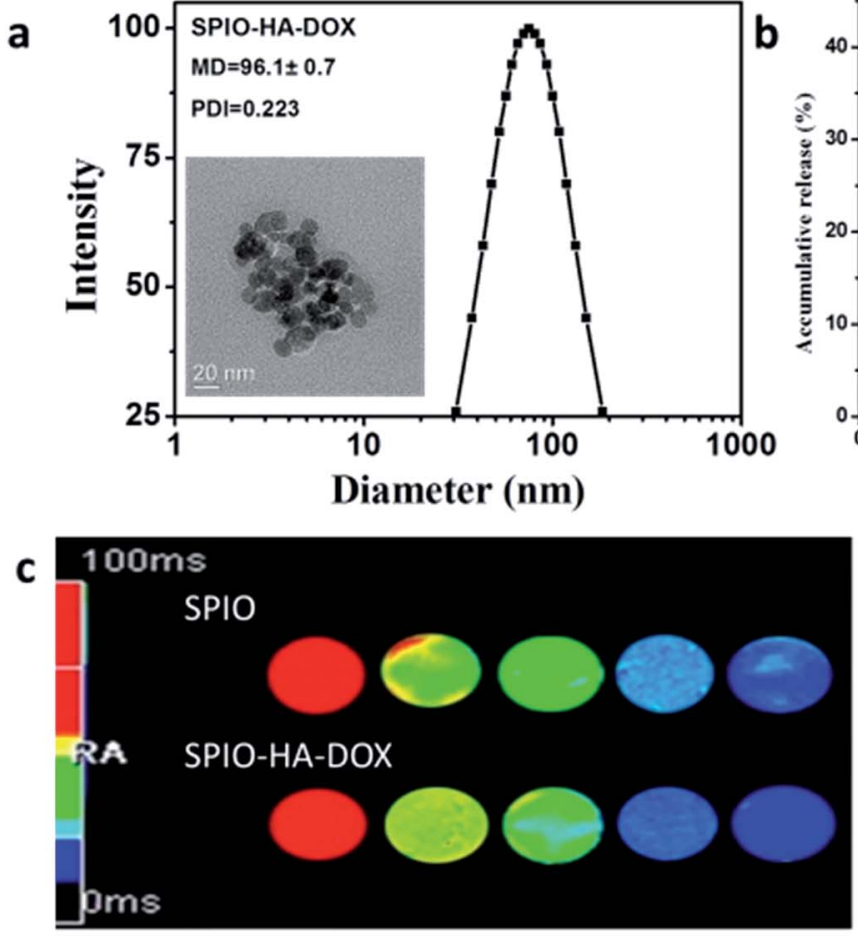
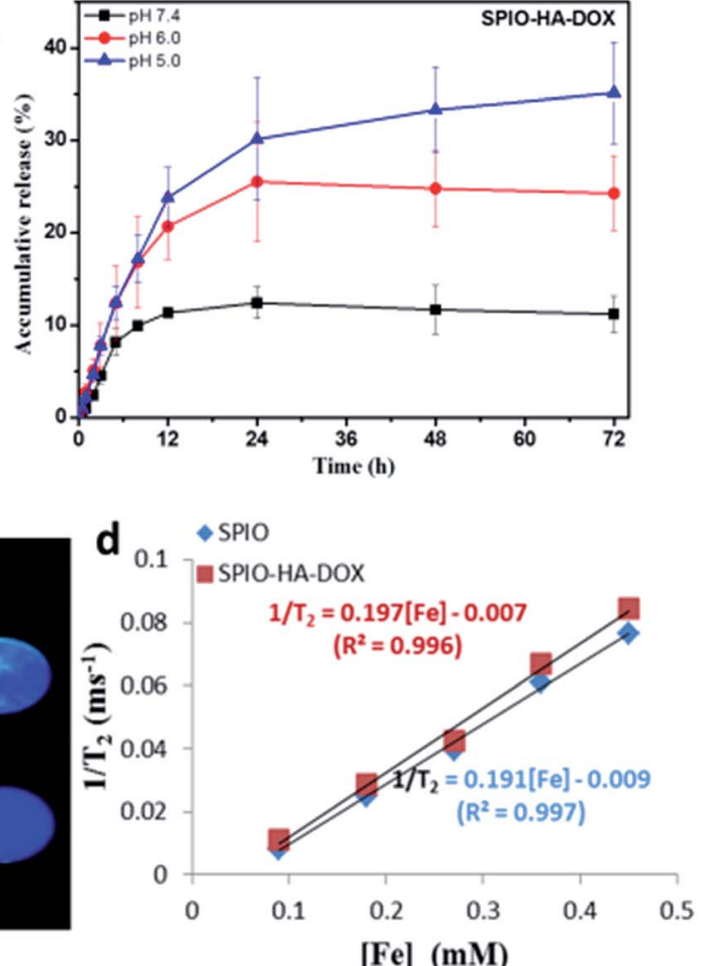

Fig. 2 DLS and TEM testing results of the SPIO-HA-DOX NPs (a), in vitro release profiles of doxorubicin from SPIO-HA-DOX in different pH (b), $T_{2}$-weighted MR images (c) and linear fitting of $1 / T_{2}(d)$, respectively. 
tumor volumes $(V)$ were calculated by the formula: $V=$ [length $\times($ width $) 2] / 2$. The mice were executed three days after the last injection.

\subsection{Histological analysis}

At the end of the in vivo MR imaging, the mice were sacrificed. Tumors were resected and quickly frozen. Frozen tumor tissue slices were fixed in acetone for $10 \mathrm{~min}$, dried in the air for $30 \mathrm{~min}$. Tumor sections (thickness: $4 \mu \mathrm{m}$ ) were then stained with Prussian blue according to standard clinical pathology protocols. In addition, at the end of the in vivo anti-tumor efficacy experiment, all of the mice in each group were sacrificed, and the tumor, heart, liver, spleen and kidney were excised. The issues were fixed with $4 \%$ paraformaldehyde solution, embedded in paraffin, cut into 4 sections and stained with hematoxylin and eosin (H\&E). All of the specimens were observed under a Olympus BX51 optical microscope.

\section{Results and discussion}

\subsection{Characteristics of the HA-DOX coated SPIOs}

HA-ADH conjugates were synthesized via a coupling primary carboxyl group of sodium hyaluronic acid with amino group of adipic acid dihydrazide as schematically shown in Fig. 1. HADOX conjugates were synthesized via a $\mathrm{pH}$-sensitive hydrazone bond formation between amine groups of HA-ADH and carbonyl of DOX. The successful synthesis of HA-DOX conjugates were confirmed by ${ }^{1} \mathrm{H}$ NMR (Fig. S2 $\dagger$ ). Fig. S3† gives the IR spectra of $\mathrm{NH}_{2}$-SPIOs and SPIO-HA-DOX. In comparison with the spectrum of $\mathrm{NH}_{2}$-SPIOs the spectrum of SPIO-HA-DOX showed not only the characteristic bands of SPIOs at 636 and $570 \mathrm{~cm}^{-1}$ (attributed to Fe-O stretching ${ }^{16}$ ) but also the characteristic band of DOX. It is showed a characteristic band of the carbonyl group at the 13-keto position at $1750 \mathrm{~cm}^{-1}$, bands at 1615 and $1586 \mathrm{~cm}^{-1}$ due to the stretching vibration of two carbonyl groups of the anthracene ring of the DOX molecule, and the bands at $998 \mathrm{~cm}^{-1}$ due to the stretching vibration of $\mathrm{C}-\mathrm{O}$ bonds. And the new bands at $1646 \mathrm{~cm}^{-1}$ and $1780 \mathrm{~cm}^{-1}$, which could be assigned to asymmetric carbonyl stretching vibration and the symmetrical stretching of carboxylic group attached to HA. ${ }^{18}$ These facts confirmed the surface modification of SPIOs by HA-DOX conjugates. The content of HA-DOX conjugates was determined to be about $13 \%$ by thermogravimetric analysis (Fig. $\mathrm{S} 4 \dagger$ ). From Fig. S6, $\dagger$ the saturation magnetization was found to be $78.1 \mathrm{emu} \mathrm{g}^{-1}$ for SPIOs and $39.3 \mathrm{emu} \mathrm{g}^{-1}$ for SPIO-HA-DOX. The mean particle size of SPIOHA-DOX NPs were estimated to be circa about $96 \mathrm{~nm}$ according to the DLS analysis. From the TEM observation, the resultant SPIO-HA-DOX were found to have an approximately spherical shape and the mean diameter was about $20 \mathrm{~nm}$, as shown in Fig. 2a. Moreover, the magnetization curves in two cases exhibit nearly zero remanence. These facts revealed that the surface modification of SPIOs by HA-DOX conjugates did not obviously affect their phase structure and superparamagnetic property.
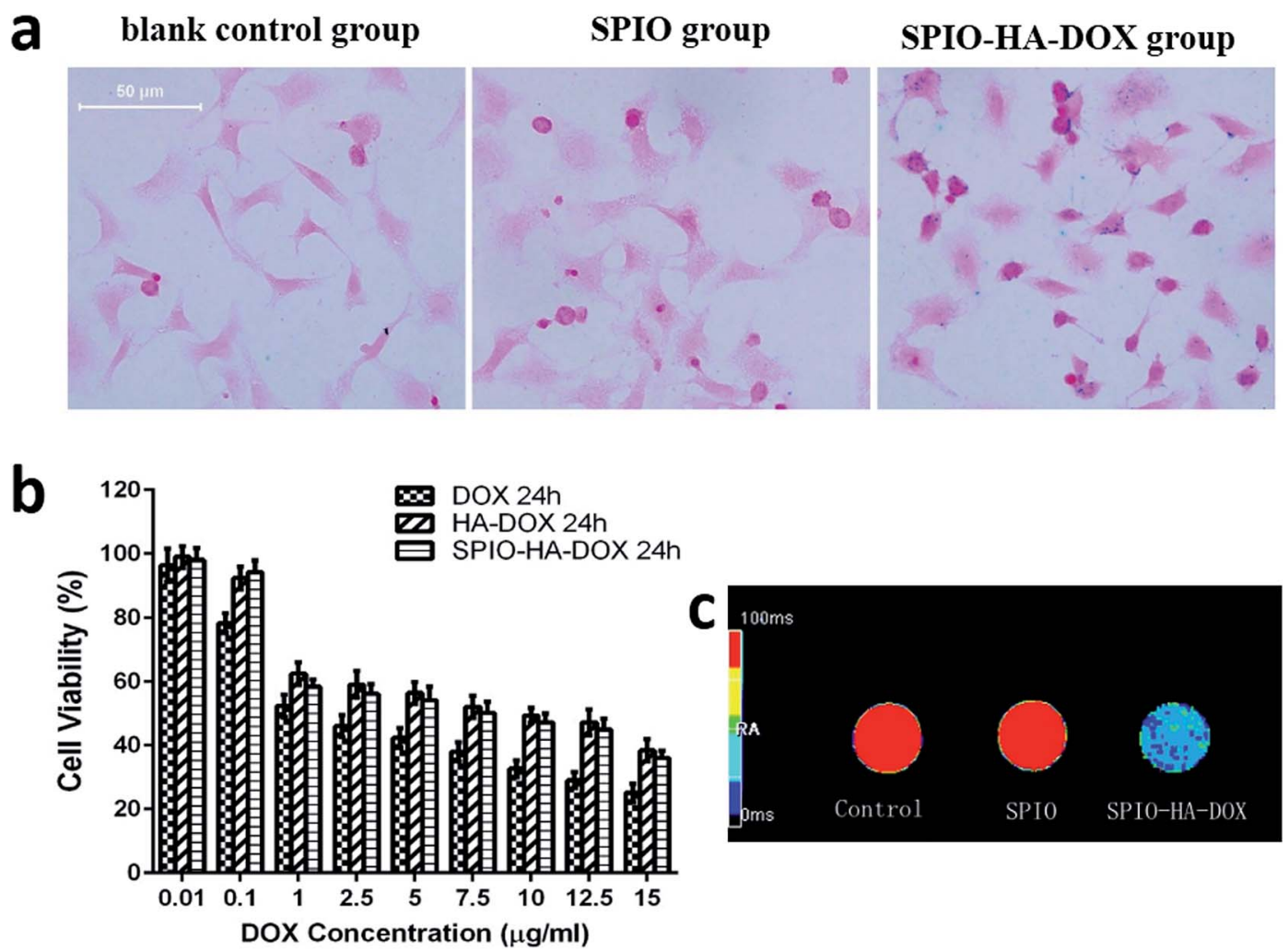

Fig. 3 In vitro Prussian blue staining images (a), in vitro cytotoxicity assays. The viability of HepG2 at $24 \mathrm{~h}$ after incubation with free DOX, HADOX and SPIO-HA-DOX at DOX concentrations ranging from 0.01 to $10 \mu \mathrm{g} \mathrm{mL}^{-1}$. (b) In vitro MR imaging (c). 


\subsection{In vitro release and $T_{2}$ relaxivity}

The in vitro release behaviors for SPIO-HA-DOX were investigated at $37{ }^{\circ} \mathrm{C}$ in different $\mathrm{pH}$ such as $\mathrm{pH} 7.4, \mathrm{pH} 6.0$ phosphate buffer solutions and acetate buffer ( $\mathrm{pH}$ 5.0), as shown in Fig. 2 b. These conjugate nanoparticles exhibited a sustained and $\mathrm{pH}$ sensitive drug release behavior. For example, the cumulative drug release at $24 \mathrm{~h}$ was found to be $30 \%$ in $\mathrm{pH} 5.0,24 \%$ in $\mathrm{pH}$ 6.0 and $11 \%$ in $\mathrm{pH} 7.4$, respectively. Similar result was also found by She et al. ${ }^{19}$ when they studied the in vitro drug release behavior of dendronized heparin DOX conjugates with the same acid-labile hydrazone linkage. In this work, DOX was combined to the conjugated by a hydrazone linkage rather than physical encapsulation, which is used amphiphilic block copolymers assembled into micellar or polymersomes structures. Thus, this conjugate may be more stable in blood circulation and the drugs may not leak out of the nanocarriers because the drug is covalently linked to the hyaluronic acid. The rate of drug release depends on the $\mathrm{pH}$ value of the release media. Similar to SPIO, SPIO-HA-DOX NPs were observed to induce the decrease of the MR signal intensity on the $T_{2}$ mapping MR images with the increase of Fe concentration (Fig. 2c). By linear fitting of the $T_{2}$ relaxation rate $\left(1 / T_{2}\right)$ as a function of Fe concentration (Fig. $2 \mathrm{~d}$ ), the specific relaxivity value was determined to be $197 \mathrm{mM}^{-1} \mathrm{~s}^{-1}$ for SPIO-HA-DOX and $191 \mathrm{mM}^{-1} \mathrm{~s}^{-1}$ for SPIOs, respectively. Therefore, SPIO-HA-DOX NPs may be used as a good $T_{2}$ negative contrast agent for sensitive MR imaging application due to their relatively higher relaxivity value.

\subsection{In vitro cellular uptake studies}

The internalization of the SPIO-HA-DOX NPs was confirmed by Prussian blue staining, a staining method giving a characteristic blue color in the presence of ferric ions. Fig. 3a shows the entrapment of SPIO stained in intense blue color into the cells. Obviously, there was no blue color appearance in cells of the control (A) and SPIO (B) group. Conversely, cells incubated with SPIO-HA-DOX NPs (Fe concentration $=40 \mu \mathrm{g} \mathrm{mL} \mathrm{mL}^{-1}$ ) were stained in intensive blue color. Consistent with previous publications, blue areas or spots could be seen in almost every cell. ${ }^{20}$ Receptor-mediated endocytosis, especially an interaction between HA and the CD44 receptor, was identified as the principal cellular uptake mechanism of the HA-based selfassembled nanoparticle. ${ }^{21,22}$ Fig. 4a exhibited the intracellular accumulation and distribution of DOX entrapped by HepG2 using fluorescence microscopy. Nuclei of cells were labelled using DAPI staining (blue fluorescence) and DOX with its natural fluorescence (red fluorescence) to follow the drug into cells. The result showed that the red and blue fluorescence were co-localized indicating that DOX was be able to reach to the nucleus which is its site of action, as reported previously. ${ }^{23} \mathrm{HA}-$ DOX and SPIO-HA-DOX group showed stronger intense intracellular fluorescence than free DOX group, confirming the cellular internalization of HA-DOX and SPIO-HA-DOX. Fig. 4b showed the flow cytometric data of different DOX formulations following incubation with HepG2 cells for $4 \mathrm{~h}$ at $37^{\circ} \mathrm{C}$. Peaks for free DOX (blue line), HA-DOX (green line) and SPIO-HA-DOX (red line) were shifted to the higher fluorescence region a
Free DOX

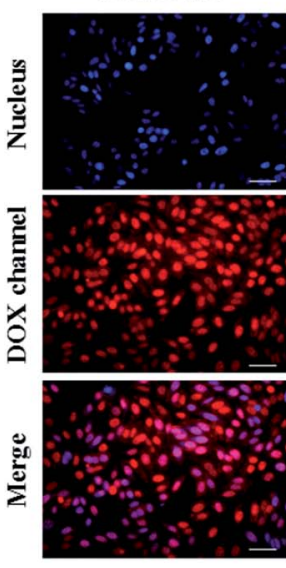

HA-DOX

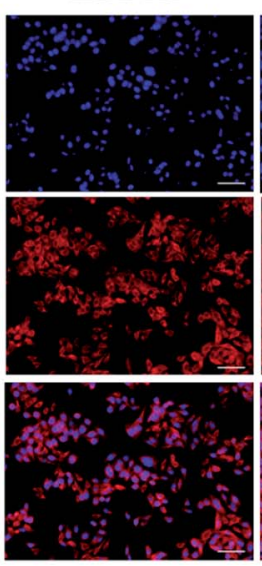

SPIO-HA-DOX

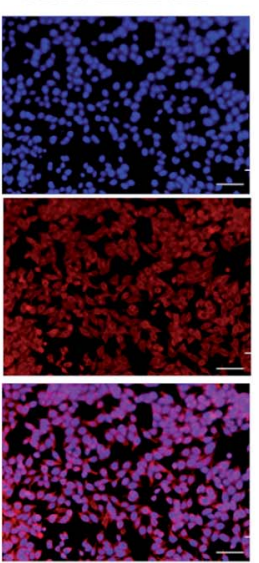

b

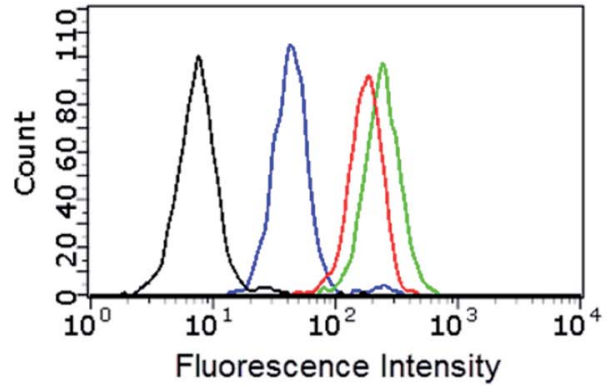

black line: control

blue line: DOX

green line: HA-DOX

red line: SPIO-HA-DOX

Fig. 4 Fluorescence microscopy images of HepG2 cells incubated with free DOX solution, HA-DOX, and SPIO-HA-DOX, respectively (a). Flow cytometry assay of DOX fluorescence intensity in HepG2 cells $\left(5 \mu \mathrm{g} \mathrm{mL}^{-1}\right)$ at $37^{\circ} \mathrm{C}$ for $4 \mathrm{~h}$ (b). Scale bar $=100 \mu \mathrm{m}$.

relative to the control (black line). In addition, the cellular fluorescence intensities of HA-DOX group and SPIO-HA-DOX group were 4.38 and 3.79 times, respectively, than DOX group, suggesting that HA-DOX and SPIO-HA-DOX evidently bound to HepG2 cells.

\subsection{In vitro cytotoxicity}

The in vitro cytotoxic effect of the DOX and SPIO-HA-DOX was evaluated by the MTT assay using HepG2 cells. The antiproliferative effect was confirmed based on previous studies with DOX in cancer cell lines. ${ }^{24}$ As shown in Fig. 3 b, the viability of tumor cells was dose-dependent. HA-DOX and SPIO-HA-DOX exhibited the inhibited ability of HepG2 cells as well as the free DOX. However, free DOX showed a higher cytotoxicity $\left(\mathrm{IC}_{50}=\right.$ $\left.1.861 \mu \mathrm{g} \mathrm{mL}{ }^{-1}\right)$ than HA-DOX $\left(\mathrm{IC}_{50}=10.236 \mu \mathrm{g} \mathrm{mL}{ }^{-1}\right)$ and SPIO-HA-DOX $\left(\mathrm{IC}_{50}=7.350 \mu \mathrm{g} \mathrm{mL}{ }^{-1}\right)$ in vitro, indicating that the SPIO-HA-DOX have significantly reduced the cytotoxicity of DOX.

\subsection{In vitro $\mathrm{MR}$ imaging}

The use of SPIO-HA-DOX NPs as the probe for the in vitro MR imaging of HepG2 cells was investigated. SPIO NPs were also 
a
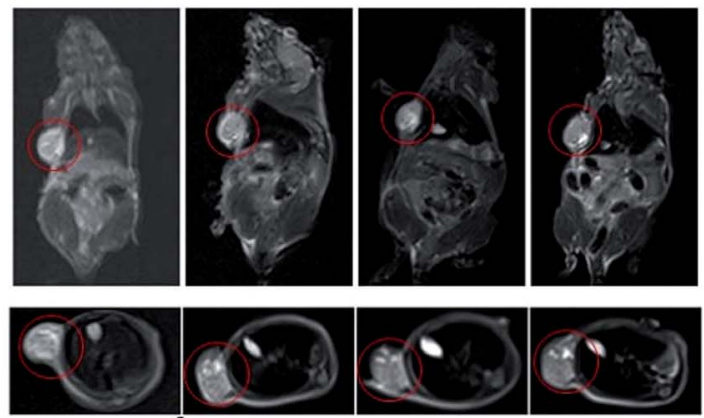

Preinjection

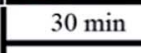

$90 \mathrm{~min}$ SPIO Post-injection

C

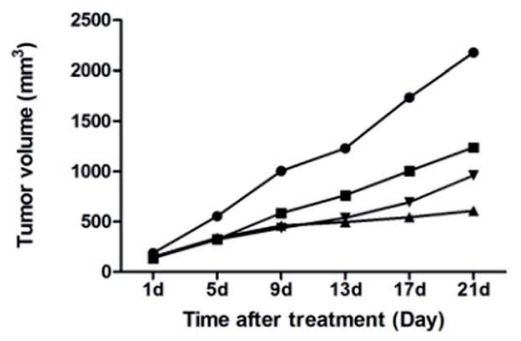

$d$

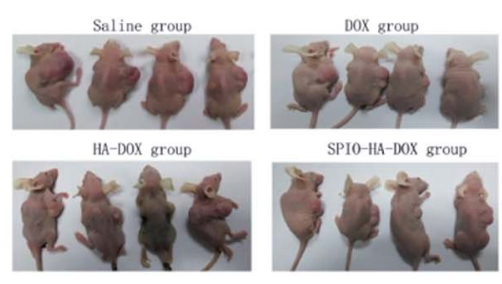

- Control Group

$\rightarrow$ DOX Group

$\star$ HA-DOX Group

* SPIO-HA-DOX Group

e
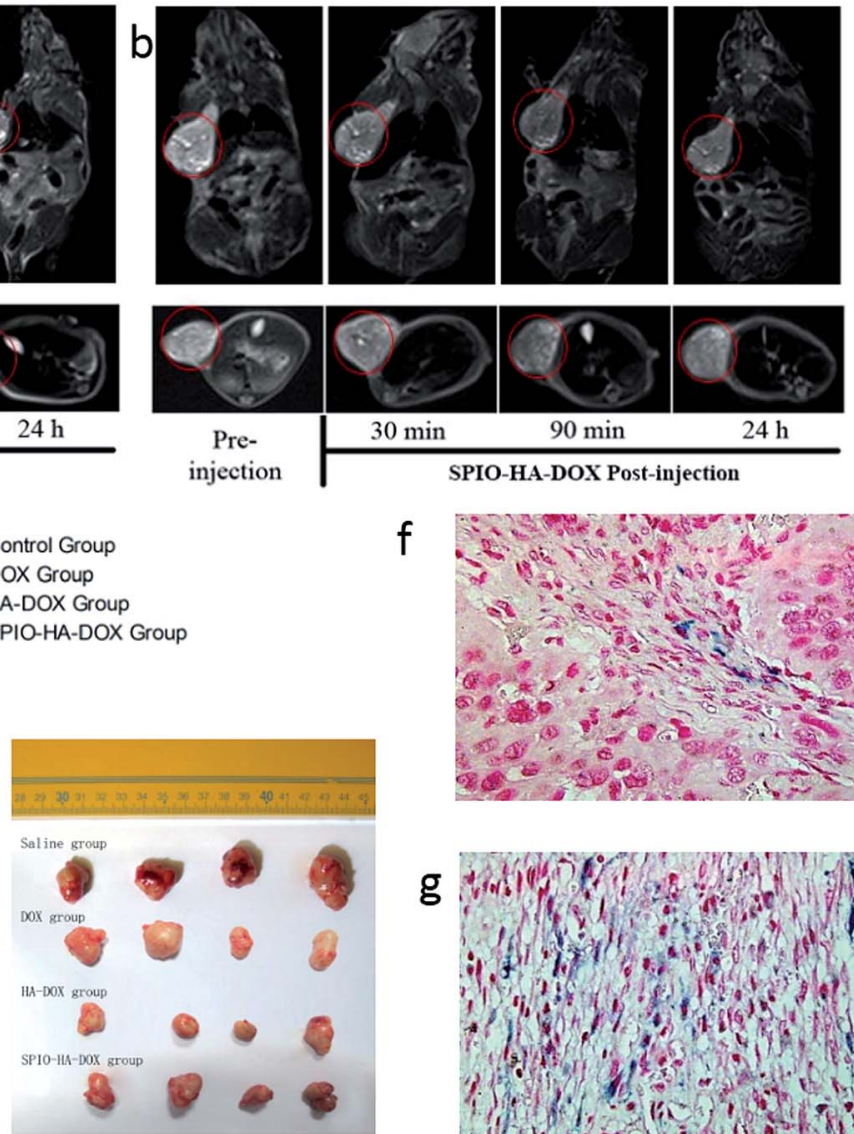

Preinjection
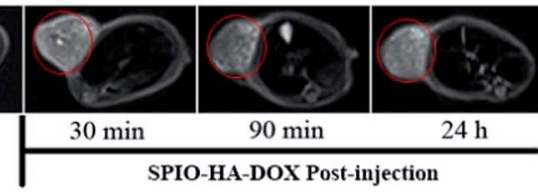

$90 \mathrm{~min}$

f

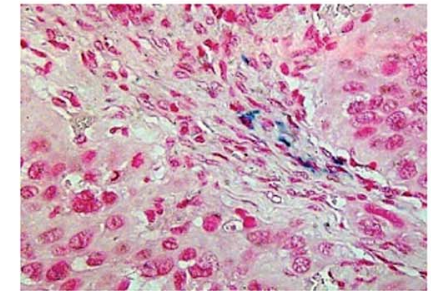

g

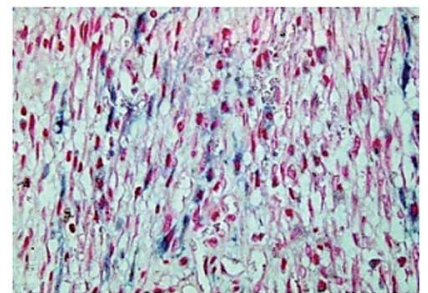

DOX group
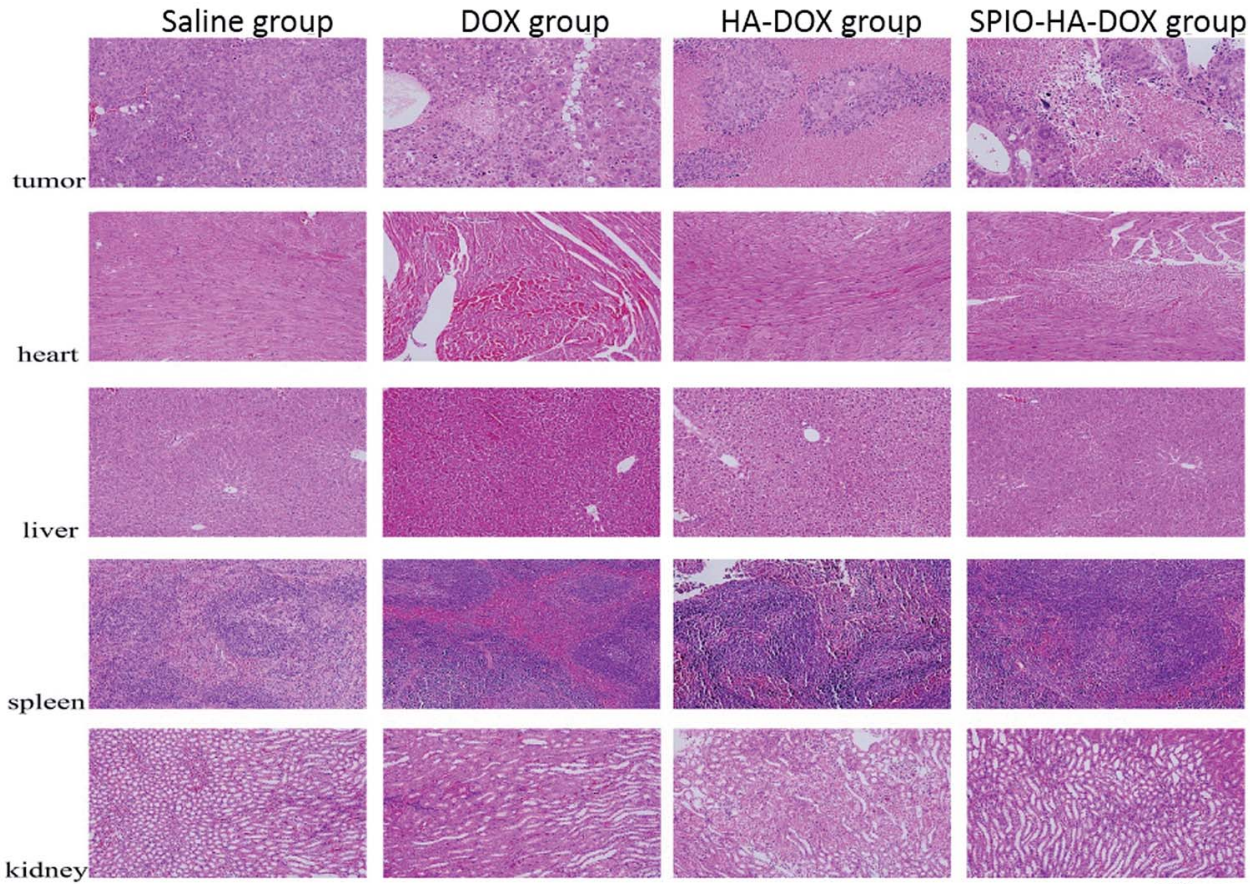

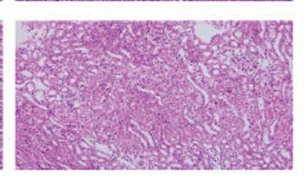

Fig. 5 In vivo MR imaging of SPIO group (a) SPIO-HA-DOX group (b); growth of HepG2 subcutaneous xenografted tumors after various treatments (c); the average tumor volume of each group at the end of the experiment ( $d$ and e); Prussian blue staining of SPIO group (f); SPIOHA-DOX group (g); representative H\&E staining images of HepG2 tumor, heart, liver, spleen and kidney on day 21 , respectively (h). 
investigated similarly for comparison. Fig. 3c gives aqueous suspension appearance, $T_{2}$ mapping images of HepG2 cells after the treatment with SPIO-HA-DOX NPs or SPIO NPs for $24 \mathrm{~h}$. For the HepG2 cells treated with SPIO-HA-DOX NPs, the in vitro MR signal intensity was found to have an obvious decrease when compared to those of untreated HepG2 cells (control group) or the cells treated with SPIO NPs. These results indicated that the accumulation of the SPIO-HA-DOX NPs was specifically mediated by the HA-CD44 binding. In contrast, SPIO NPs without HA modification did not result in an appreciable change of the MR signal intensity.

\subsection{In vivo MR imaging}

As is acknowledged, direct imaging of cancers using modified SPIO NPs have shown great potential in the biomedical field. ${ }^{25,26}$ Thus, we explored the use of SPIO-HA-DOX NPs as an MRI contrast agent for in vivo cancer detection, and unmodified SPIOs NPs conjugates as control. Tumors are seen as hyperintense areas in $T_{2}$-weighted $\mathrm{MR}$ images at $0 \mathrm{~h}$ prior to the injection, as indicated by the red circles in Fig. $5 \mathrm{a}$ and $\mathrm{b}$. The heterogeneous signal of tumor, as the previous report, was attributed to hemorrhage, necrosis, cystic formation. ${ }^{27}$ For both of the two groups, we could see an appreciable darkening of $T_{2}$-weighted MR images from the tumor areas at post-injection relative to pre-injection. The tumor showed an average signal intensity decrease of $9.8 \%$ at 0.5 hour after injecting SPIO-HADOX NPs, while only $4.9 \%$ decrease in the SPIO group. Moreover, at 1.5 hours after injection, the $T_{2}$ signal drop for SPIO-HADOX NPs was as high as $28.9 \%$, suggesting an ideal post injection time window for MRI scanning. By contrast, the $T_{2}$ signal drop for SPIO NPs was only $8.1 \%$ at this time point. The MRI $T_{2}$ signal intensity was slowly recovered, along with further extending post injection time for MRI measurements. It indicated that, the targeting ligand HA facilitated the delivery of
SPIO NPs into tumor cells, and thus rendering targeted SPIO NPs superior than SPIO NPs delivered only through the enhanced permeation and retention (EPR) effect.

\subsection{In vivo anti-tumor efficacy}

Based on the low in vitro cytotoxic activity of SPIO-HA-DOX against hepatic carcinoma cells, the in vivo anti-tumor activity in the HepG2 subcutaneous tumor model was also evaluated. Fig. $5 \mathrm{c}$ and $\mathrm{d}$ showed the results of in vivo anti-tumor efficacy. Compared with the control group, all treatment containing DOX (free DOX; HA-DOX; SPIO-HA-DOX) dramatically reduced tumor growth $(P<0.05)$ and induced a higher delay in tumor growth. Interestingly, for the various DOX formulations, the free DOX exhibited the least inhibition of tumor growth, which was inconsistent with the results of our in vitro cytotoxicity studies. It is speculated that it might resulted from the free DOX's short circulation time and absence of specific tumor targeting. Additionally, our experiment showed HA-DOX inhibited tumor growth more effectively than SPIO-HA-DOX, which indicated the former has the longer duration than the latter agent. Overall, these results suggested that SPIO-HA-DOX NPs possessed high anti-tumor activity and had the potential as the theranostic agent for cancer diagnostic and treatment.

\subsection{Histological analysis}

To further verify the accumulation of SPIO-HA-DOX NPs in the tumor tissue, Prussian blue staining was carried out, as shown in Fig. 5g. Blue spots of SPIO-HA-DOX showed tumor cells' localization. In non-targeted group (Fig. 5f), only a little SPIO NPs was observed with an unspecific distribution. The tumor inhibition efficacy was confirmed via H\&E staining of tumor sections. For saline-treated group, well-defined tumor tissues stained in blue can be clearly seen throughout the whole slice.

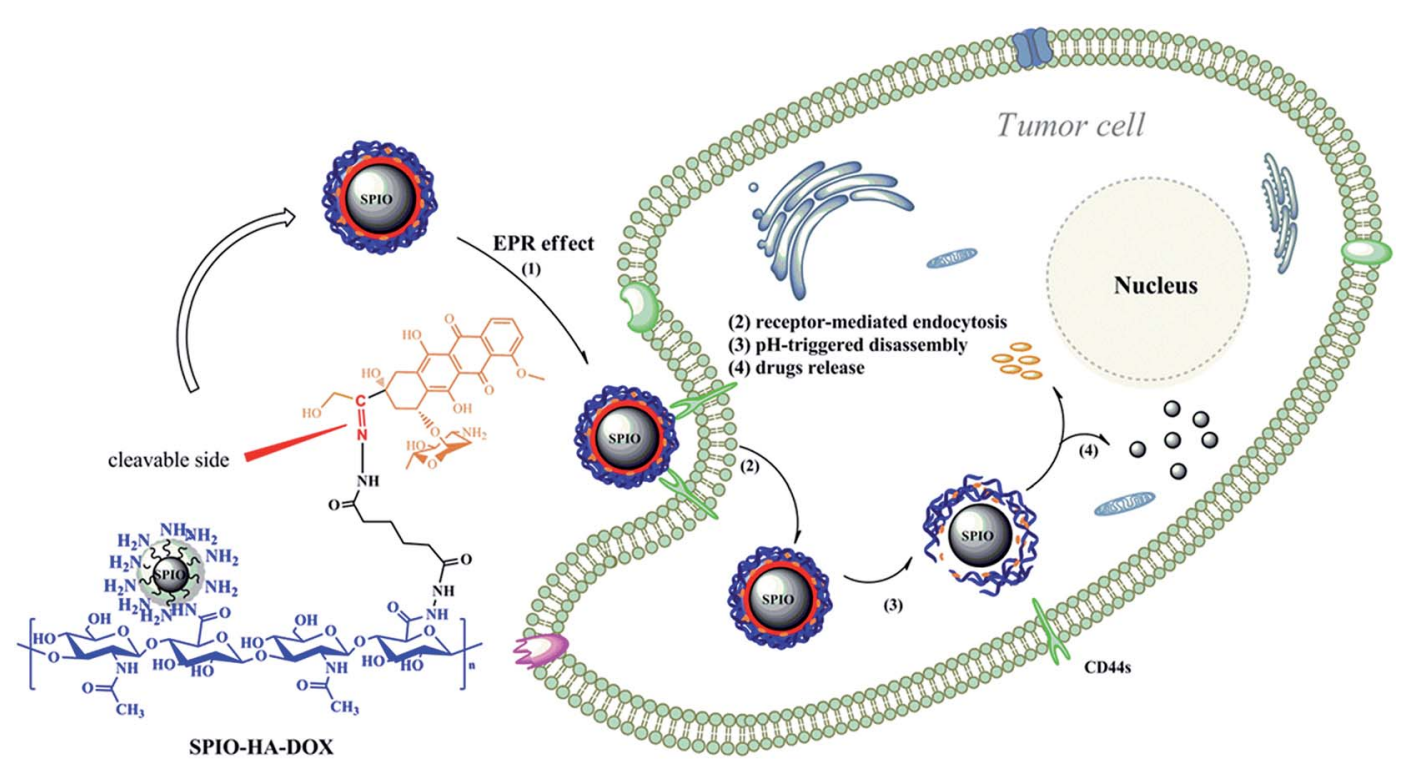

Scheme 1 Illustration of the formation, receptor-mediated endocytosis and $\mathrm{pH}$-stimuli responsive drug and SPIO release of SPIO-HA-DOX nanoparticles. 
In the DOX-treated group, a large portion of tumor tissue and a small portion of necrosis could be seen, demonstrating the inhibition efficacy of free DOX. As opposed to saline and free DOX group, in the tumor section treated by HA-DOX and SPIOHA-DOX group, a majority of necrotic tissue stained in pink and only a small portion of tumor tissue stained in blue could be seen. The clear histological differences further demonstrated the enhanced therapeutic efficacy of SPIO-HA-DOX. At 21 days post treatment, all the mice treated with saline, free DOX, HASPIO and SPIO-HA-DOX were sacrificed, and the major organs including the heart, liver, spleen and kidney were collected for pathological examination via H\&E staining (Fig. 5h). All of the studied organs from the mice treated with HA-DOX or SPIO-HADOX showed no noticeable damage when compared to the saline group, suggesting a good biocompatibility of SPIO-HADOX, as well as HA-DOX. Therefore, SPIO-HA-DOX can be safely used in drug delivery system.

\section{Conclusions}

pH responsive prodrug and SPIO NPs co-delivery systems hold promise to combinatorial monitoring and chemotherapy of liver carcinoma. In the current study, we successfully synthesized surface functionalization of superparamagnetic nanoparticles by an acid-liable polysaccharide-based prodrug SPIOHA-DOX, for the first time, by conjugating HA-DOX and SPIOs through a carbodiimide mediated coupling reaction. The surface modification of SPIO nanoparticles with a stable diameter of about $100 \mathrm{~nm}$. These NPS had no initial burst release, but exhibited sustained and $\mathrm{pH}$-responsive release behavior. Furthermore, SPIO-HA-DOX NPs exhibited exquisite ability to targeted MRI and highly effective chemotherapy for CD44 HA receptors highly expressing liver cancer in both in vitro and in vivo studies. The surface functionalization of magnetic iron oxide with stimuli responsive polysaccharide-based prodrugs may be well utilized as an efficient strategy in clinical cancer theranostic field (Scheme 1).

\section{Conflicts of interest}

There are no conflicts of interest to declare.

\section{Acknowledgements}

This work is supported by the 973 Program of China (2015CB755500), the National Natural Science Foundation of China (51273216, 81571665, 21074152, J1103305), Guangzhou Key Technology R\&D Program (1563000477, 201607010038), and the Natural Science Foundation of Guangdong Province in China (20153100042020850, 10151006001000010, 2014A030313647, 2014A010105025), Social Development Program Fund of Guangdong Province (2014A020212031).

\section{References}

1 A. M. Fales, H. Yuan and T. Vo-Dinh, Silica-coated gold nanostars for combined surface-enhanced Raman scattering (SERS) detection and singlet-oxygen generation: a potential nanoplatform for theranostics, Langmuir, 2016, 27(19), 12186-12190.

2 S. S. Kelkar and T. M. Reineke, Theranostics: combining imaging and therapy, Bioconjugate Chem., 2011, 22(10), 1879-1903.

3 H. Kim, K. Chung, S. Lee, D. H. Kim and H. Lee, Nearinfrared light-responsive nanomaterials for cancer theranostics, Wiley Interdiscip. Rev.: Nanomed. Nanobiotechnol., 2016, 1(8), 23-45.

4 Y. Lyu, Y. Fang, Q. Miao, X. Zhen, D. Ding and K. Pu, Intraparticle Molecular Orbital Engineering of Semiconducting Polymer Nanoparticles as Amplified Theranostics for in Vivo Photoacoustic Imaging and Photothermal Therapy, ACS Nano, 2016, 10(4), 4472-4481.

5 T. Krasia-Christoforou and T. K. Georgiou, Polymeric theranostics: using polymer-based systems for simultaneous imaging and therapy, J. Mater. Chem. B, 2013, 1(24), 3002-3025.

6 R. Cheng, F. Meng, C. Deng, H. A. Klok and Z. Zhong, Dual and multi-stimuli responsive polymeric nanoparticles for programmed site-specific drug delivery, Biomaterials, 2013, 34(14), 3647-3657.

7 T. Boissenot, E. Fattal, A. Bordat, S. Houvenagel, J. Valette, H. Chacun, C. Gueutin and N. Tsapis, Paclitaxel-loaded PEGylated nanocapsules of perfluorooctyl bromide as theranostic agents, Eur. J. Pharm. Biopharm., 2016, 108, 136-144.

8 W. Chen, N. Xu, L. Xu, L. Wang, Z. Li, W. Ma, Y. Zhu, C. Xu and N. A. Kotov, Multifunctional magnetoplasmonic nanoparticle assemblies for cancer therapy and diagnostics (theranostics), Macromol. Rapid Commun., 2010, 31(2), 228236.

9 Y. Zhong, M. Dimde, D. Stöbener, F. Meng, C. Deng, Z. Zhong and R. Haag, Micelles with Sheddable Dendritic Polyglycerol Sulfate Shells Show Extraordinary TumorTargetability and Chemotherapy in Vivo, ACS Appl. Mater. Interfaces, 2016, 8(41), 27530-27538.

10 J. H. Ryu, S. Lee, S. Son, S. H. Kim, J. F. Leary, K. Choi and I. C. Kwon, Theranostic nanoparticles for future personalized medicine, J. Controlled Release, 2014, 190, 477-484.

11 Z. Gu, L. Yan, G. Tian, S. Li, Z. Chai and Y. Zhao, Recent advances in design and fabrication of upconversion nanoparticles and their safe theranostic applications, $A d v$. Mater., 2013, 25(28), 3758-3779.

12 S. Ruan, M. Yuan, L. Zhang, G. Hu, J. Chen, X. Cun, Q. Zhang, Y. Yang, Q. He and H. Gao, Tumor microenvironment sensitive doxorubicin delivery and release to glioma using angiopep-2 decorated gold nanoparticles, Biomaterials, 2015, 37, 425-435.

13 M. Yu, S. Jambhrunkar, P. Thorn, J. Chen, W. Gu and C. Yu, Hyaluronic acid modified mesoporous silica nanoparticles for targeted drug delivery to CD44-overexpressing cancer cells, Nanoscale, 2013, 5(1), 178-183.

14 R. Yang, C. Fu, N. Li, L. Wang, X. Xu, D. Yang, J. Fang, $\mathrm{X}$. Jiang and L. Zhang, Glycosaminoglycan-targeted iron 
oxide nanoparticles for magnetic resonance imaging of liver carcinoma, Mater. Sci. Eng., C, 2014, 45, 556-563.

15 X. Jia, G. Colombo, R. Padera, R. Langer and D. S. Kohane, Prolongation of sciatic nerve blockade by in situ crosslinked hyaluronic acid, Biomaterials, 2004, 25(19), 47974804.

16 J. Mürbe, A. Rechtenbach and J. Töpfer, Synthesis and physical characterization of magnetite nanoparticles for biomedical applications, Mater. Chem. Phys., 2008, 110(2), 426-433.

17 E. J. van den Bos, A. Wagner, H. Mahrholdt, R. B. Thompson, Y. Morimoto, B. S. Sutton, R. M. Judd and D. A. Taylor, Improved efficacy of stem cell labeling for magnetic resonance imaging studies by the use of cationic liposomes, Cell Transplant., 2003, 12(7), 743-756.

18 Y. Chang, X. Meng, Y. Zhao, K. Li, B. Zhao, M. Zhu, Y. Li, $\mathrm{X}$. Chen and J. Wang, Novel water-soluble and $\mathrm{pH}-$ responsive anticancer drug nanocarriers: doxorubicinPAMAM dendrimer conjugates attached to superparamagnetic iron oxide nanoparticles (IONPs), $J$. Colloid Interface Sci., 2011, 363(1), 403-409.

19 W. She, N. Li, K. Luo, C. Guo, G. Wang, Y. Geng and Z. Gu, Dendronized heparin- doxorubicin conjugate based nanoparticle as $\mathrm{pH}$-responsive drug delivery system for cancer therapy, Biomaterials, 2013, 34(9), 2252-2264.

20 M. H. El-Dakdouki, D. C. Zhu, K. El-Boubbou, M. Kamat, J. Chen, W. Li and X. Huang, Development of multifunctional hyaluronan-coated nanoparticles for imaging and drug delivery to cancer cells, Biomacromolecules, 2012, 13(4), 1144-1151.

21 H. J. Cho, H. Y. Yoon, H. Koo, S. H. Ko, J. S. Shim, J. H. Cho, J. H. Park, K. Kim and D. D. Kim, Hyaluronic acid-ceramide- based optical/MR dual imaging nanoprobe for cancer diagnosis, J. Controlled Release, 2012, 162(1), 111-118.

22 W. I. Choi, J. Y. Kim, S. U. Heo, Y. Y. Jeong, Y. H. Kim and G. Tae, The effect of mechanical properties of iron oxide nanoparticle-loaded functional nano-carrier on tumor targeting and imaging, J. Controlled Release, 2012, 162(2), 267-275.

23 N. Schleich, P. Sibret, P. Danhier, B. Ucakar, S. Laurent, R. N. Muller, C. Jérôme, B. Gallez, V. Préat and F. Danhier, Dual anticancer drug/superparamagnetic iron oxide-loaded PLGA-based nanoparticles for cancer therapy and magnetic resonance imaging, Int. J. Pharm., 2013, 447(1-2), 94-101.

24 Y. Chang, N. Liu and L. Chen, Synthesis and characterization of DOX-conjugated dendrimer-modified magnetic iron oxide conjugates for magnetic resonance imaging, targeting, and drug delivery, J. Mater. Chem., 2012, 22(19), 9594-9601.

25 D. Frascione, C. Diwoky, G. Almer, P. Opriessnig, C. Vonach, K. Gradauer, G. Leitinger, H. Mangge, R. Stollberger and R. Prassl, Ultrasmall superparamagnetic iron oxide (USPIO)-based liposomes as magnetic resonance imaging probes, Int. J. Nanomed., 2012, 7, 2349-2359.

26 G. Hong, R. Yuan, B. Liang, J. Shen, X. Yang and X. Shuai, Folate-functionalized polymeric micelle as hepatic carcinoma-targeted, MRI-ultrasensitive delivery system of antitumor drugs, Biomed. Microdevices, 2008, 10(5), 693-700.

27 H. Y. Yoon, G. Saravanakumar, R. Heo, S. H. Choi, I. C. Song, M. H. Han, K. Kim, J. H. Park, K. Choi, I. C. Kwon and K. Park, Hydrotropic magnetic micelles for combined magnetic resonance imaging and cancer therapy, $J$. Controlled Release, 2012, 160(3), 692-698. 